\title{
ClinicalTrials.gov registration can supplement information in abstracts for systematic reviews: a comparison study
}

\author{
Roberta W Scherer ${ }^{*}$, Lynn Huynh", Ann-Margret Ervin ${ }^{1}$, Jakeisha Taylor ${ }^{2}$ and Kay Dickersin ${ }^{1}$
}

\begin{abstract}
Background: The inclusion of randomized controlled trials (RCTs) reported in conference abstracts in systematic reviews is controversial, partly because study design information and risk of bias is often not fully reported in the abstract. The Association for Research in Vision and Ophthalmology (ARVO) requires trial registration of abstracts submitted for their annual conference as of 2007. Our goal was to assess the feasibility of obtaining study design information critical to systematic reviews, but not typically included in conference abstracts, from the trial registration record.

Methods: We reviewed all conference abstracts presented at the ARVO meetings from 2007 through 2009, and identified 496 RCTs; 154 had a single matching registration record in ClinicalTrials.gov. Two individuals independently extracted information from the abstract and the ClinicalTrials.gov record, including study design, sample size, inclusion criteria, masking, interventions, outcomes, funder, and investigator name and contact information. Discrepancies were resolved by consensus. We assessed the frequencies of reporting variables appearing in the abstract and the trial register and assessed agreement of information reported in both sources.
\end{abstract}

Results: We found a substantial amount of study design information in the ClinicalTrials.gov record that was unavailable in the corresponding conference abstract, including eligibility criteria associated with gender (83\%;

128/154); masking or blinding of study participants (53\%, 82/154), persons administering treatment (30\%, 46/154), and persons measuring the outcomes (40\%, 61/154)); and number of study centers (58\%; 90/154). Only 34\%

(52/154) of abstracts explicitly described a primary outcome, but a primary outcome was included in the "Primary Outcome" field in the ClinicalTrials.gov record for 82\% (126/154) of studies. One or more study interventions were reported in each abstract, but agreed exactly with those reported in ClinicalTrials.gov only slightly more than half the time $(88 / 154,56 \%)$. We found no contact information for study investigators in the abstract, but this information was available in less than one quarter of ClinicalTrial.gov records (17\%; 26/154).

Conclusion: RCT design information not reported in conference abstracts is often available in the corresponding ClinicalTrials.gov registration record. Sometimes there is conflicting information reported in the two sources and further contact with the trial investigators may still be required.

\section{Background}

Identification of relevant randomized controlled trials (RCTs) is an integral part in the conduct of systematic reviews of intervention efficacy and effectiveness. RCTs are usually identified through searching electronic databases (e.g., PubMed, EMBASE) and handsearching (manually screening biomedical journals, conference proceedings

\footnotetext{
* Correspondence: rscherer@jhsph.edu

${ }^{1}$ Center for Clinical Trials, Department of Epidemiology, Johns Hopkins

Bloomberg School of Public Health, Baltimore, MD 21205, USA

Full list of author information is available at the end of the article
}

and other publications). Recently, the Institute of Medicine (IOM) published guidelines stating that results from conference abstracts should be included in systematic reviews because conference abstracts provide an important source of unpublished trials [1]. This is because only about $60 \%$ of controlled clinical trials presented as conference abstracts are subsequently published as journal articles, and trials with negative or null results are published less frequently than those with positive findings [2]. The end result is that inclusion of the grey literature in systematic reviews and meta-analyses leads to smaller 
treatment effect sizes overall [3]. This presents a problem for systematic reviewers, however, as reporting of study design in abstracts is poor [4] and results reported are often preliminary. Furthermore, there are concerns that abstracts rarely undergo peer review. Although adoption of CONSORT reporting guidelines for conference abstracts may lead to some improvement over time, controversy remains about whether to include conference abstracts in systematic reviews [5-7].

One approach to learning more about critical design elements of trials reported only in conference abstracts is to seek information from other sources. For example, a trials register such as ClinicalTrials.gov includes key protocol items relevant to determining eligibility and performing critical appraisal of a study.

We hypothesized that information included in a trials register record could be used to supplement the sparse information on study design presented in a conference abstract. To test the hypothesis, we obtained reports of RCTs presented at the annual meeting of the Association for Research in Vision and Ophthalmology (ARVO) from 2007 to 2009; this is an international meeting of more than 10,000 attendees. The ARVO organizers require that any abstract submitted for presentation at the annual meeting that describes a concurrently controlled trial must be registered in an electronically searchable, publicly available trials register [8]. The online conference abstract submission form includes a box in which abstract authors reporting controlled clinical trials are asked to supply the name of the trials register and the trial registration number where the trial was registered. Accompanying instructions include a definition of a clinical trial and a hyperlink to a frequently asked questions page that describes trials registers, including a drop-down menu of acceptable registers. A previous study showed that when authors complied with this requirement, about 90\% reported registering trials at ClinicalTrials.gov [9].

\section{Methods \\ Identification of included randomized controlled clinical trials}

We reviewed all abstracts presented at the ARVO meetings from 2007 through 2009 (submitted through December 2008). Abstracts were classified as RCTs using the definition provided in the Cochrane Collaboration's Handsearching Training Manual: "a study in which individuals (or other units) followed in the trial were definitely assigned prospectively to one of two (or more) alternative forms of health care using random allocation" [10]. One person hand searched the ARVO annual meeting abstracts online at http://www.arvo.org. A second person reviewed all studies classified as an RCT by the handsearcher and another person reviewed a sample of abstracts not classified as an RCT [9]. Discrepancies were resolved by consensus.

Two individuals independently extracted information about trial registration as reported for each abstract, including the name of the organization listed in the trial registration box and information in the box designated for the registration identification number. Abstracts listing more than one registration number or not listing any number were excluded from further analysis. Because $88 \%(276 / 312)$ of RCTs reporting a trials register listed ClinicalTrials.gov as the trials register [9], we chose to include only ClinicalTrials.gov records in our study. We entered the trial register number provided in the search box at http://www.clinicaltrials.gov and classified numbers as valid if a matching trial was identified in ClinicalTrials.gov, and invalid if the search yielded no results. We did not attempt to identify a ClinicalTrials.gov registration record for trials whose investigators had not included a registration number. We retrieved all ClinicalTrials.gov records for trials as posted (i.e., including amendments incorporated in the record at the time of retrieval) from May and June, 2009.

Two persons independently reviewed the abstractClinicalTrials.gov pairs for inclusion. We excluded abstract-ClinicalTrials.gov pairs that described secondary analyses of trial data (e.g., analyses of ancillary study data), nested case-control studies from RCT data, and methodological studies associated with the RCT, because the objective of our study was to compare descriptions of the original RCT design; we also excluded pairs where the ClinicalTrials.gov record stated that assignment to treatment was not randomized.

\section{Abstraction of study design characteristics}

We extracted information from both the abstract and the ClinicalTrials.gov record, including type of randomized comparison (parallel, cross over, cluster), multi-center status (yes/no), number randomized, and inclusion criteria related to demographic characteristics of the study population (i.e., adults, children, included sexes, presence of a disease or condition, and/or healthy volunteers). Masking was characterized separately as yes/no for study participants, treatment administrators, and outcome assessors. The study intervention, primary outcome, secondary outcomes, and study funder(s) were extracted verbatim from the abstract and the ClinicalTrials.gov record. We classified an outcome as the "primary" outcome in the abstract only if it was explicitly stated as such, and in the ClinicalTrials.gov record only if it was included in the "Primary Outcome" field in the tabular view of the record. We classified all other outcomes reported as "nonprimary" outcomes. The study funder was abstracted from the "Support" field of the abstract and either the "Sponsor" or "Collaborator" field in the ClinicalTrials.gov record. 
We classified funders as industry or non-industry. If the study funder was unclear, we classified it as non-industry as a conservative measure. We also collected information about study status as reported in ClinicalTrials.gov ("not yet recruiting", "recruiting", "active", or "not recruiting, completed"). If more than one abstract had the same registration number, we extracted information from all relevant abstracts and reported it on a single data abstraction form. We also extracted the presence of a contact name, telephone number, and e-mail address from the ClinicalTrials.gov record. All data were abstracted independently by two abstractors (RWS, LH, KD, AE, JT) on pre-tested paper data collection forms; we extracted all information from the abstract before we extracted information from the ClinicalTrials.gov record. Discrepancies were resolved by consensus. All data from the paper forms were entered into an Access database.

\section{Data analyses}

We assessed frequencies of reporting variables in the abstract and the trials register. We assessed the concordance between study design characteristics that were described in both the conference abstract and the trials register record by comparing reports of variables across abstract-ClinicalTrials.gov pairs. We determined whether a design characteristic that was not described in the abstract was present in the trials register. We also compared information reported in both sources and characterized the level of agreement as full, partial, or no agreement. If the abstract and the ClinicalTrials.gov record agreed exactly or nearly exactly, we classified this as full agreement. A more precise definition of an outcome in the abstract (or register) was classified as partial agreement while a completely different outcome in the abstract was classified as a new outcome. We further categorized partial agreement by type of disagreement and source of additional information (i.e., the abstract or the ClinicalTrials.gov record). We used SAS Version 9.2 (SAS Institute, Carey, NC) to perform all analyses.

\section{Results}

\section{Registration of clinical trials}

The handsearching results have previously been reported [9]. Only $2.8 \%(496 / 17,953)$ of all abstracts presented at ARVO from 2007 to 2009 described results of an RCT; 276 of these reported registration in ClinicalTrials.gov. Excluding abstracts not meeting our eligibility criteria resulted in 158 abstracts. We also excluded 4 abstracts in which the ClinicalTrials.gov record classified the study as not randomized. We linked each of the remaining abstracts with a single ClinicalTrials.gov registration number, resulting in 154 abstract-ClinicalTrials.gov pairs for analysis (see Figure 1).

\section{General study design}

We observed generally good agreement on the randomized intervention comparison described in the abstract with what was reported in ClinicalTrials.gov (80.5\%, 124/154) (see Table 1). Whether a trial was single or multi-center was the same in 39 pairs, different in 13, and not reported in either source for 12 pairs. For the remaining 90 pairs, no information on multi-center

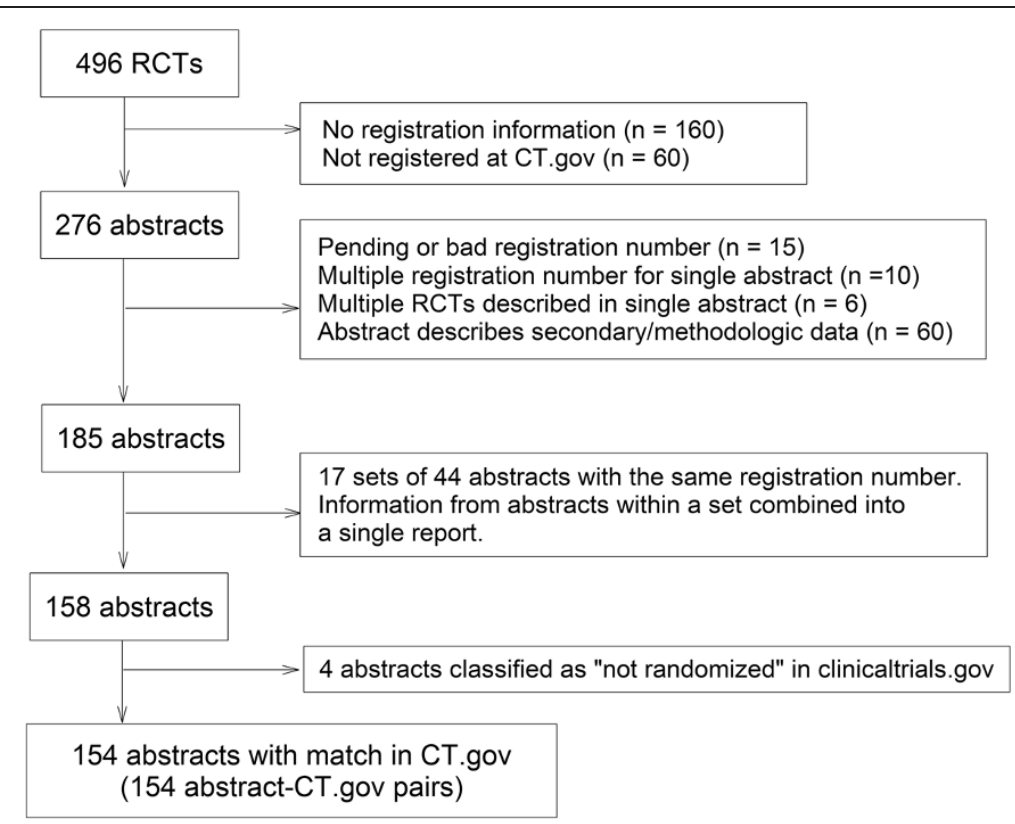

Figure 1 Flow chart of conference abstract-ClinicalTrial.gov register pairs used for comparison of randomized controlled trial characteristics. $R C T$ = randomized controlled trial; $C T$.gov = ClinicalTrials.gov. 
Table 1 Agreement between conference abstract and ClinicalTrials.gov register on design of randomized comparison ( $\mathrm{n}=154$ abstract-ClinicalTrials.gov pairs)

\begin{tabular}{lccccccc}
\hline \multirow{2}{*}{ Abstract } & \multicolumn{7}{c}{ ClinicalTrials.gov } \\
\cline { 2 - 7 } & Parallel & Cross-over & Cluster & Within person $^{*}$ & Factorial & Other & Total number of pairs $^{\text {Parallel }}$ \\
Cross-over & $\mathbf{9 7}$ & 5 & 0 & 1 & 2 & 7 & $\mathbf{1 1 2}$ \\
Cluster & 1 & $\mathbf{2 0}$ & 0 & 0 & 1 & 0 & $\mathbf{2 2}$ \\
Within person* & 0 & 0 & $\mathbf{1}$ & 0 & 0 & 0 & $\mathbf{1}$ \\
Factorial & 8 & 1 & 0 & $\mathbf{6}$ & 0 & $\mathbf{1}$ & $\mathbf{0}$ \\
Other & 0 & 0 & 0 & 0 & 0 & $\mathbf{0}$ & $\mathbf{3}$ \\
Total number of pairs & $\mathbf{1 0 8}$ & $\mathbf{2 7}$ & $\mathbf{1}$ & $\mathbf{7}$ & $\mathbf{3}$ & $\mathbf{8}$ & $\mathbf{1 5 4}$ \\
\hline
\end{tabular}

* Trials in which the unit of randomization is not the individual, but an organ or part of the body (e.g., eyes).

status was available in the abstract, but was reported in ClinicalTrials.gov.

\section{Inclusion criteria}

There was agreement between the abstract and ClinicalTrials.gov record on inclusion criteria related to age, the presence of a disease or condition, and whether the trial participants were healthy volunteers (see Figure 2). The inclusion criterion of sex was rarely reported in the abstract, but when it was reported, the information usually agreed with what was reported in ClinicalTrials.gov. In contrast, the inclusion of men and/or women was always included in the ClinicalTrials.gov record.

\section{Intervention}

At least one experimental and one control intervention was reported in both the abstract and the ClinicalTrials. gov record. We observed good agreement in the intervention reported in the abstract with that reported in ClinicalTrials.gov when looking at the broad categories of treatment, prevention, diagnostic tests, and other (Table 1). However, there was less agreement when we compared more precise descriptions of the interventions. The interventions described in the abstract and the ClinicalTrials.gov record agreed exactly for 56\% (88/154) of pairs, while $37 \%(57 / 154)$ of pairs agreed partly and $6 \%(9 / 154)$ of pairs did not agree (see Table 2 for examples of disagreements). In eight pairs, partial agreement involved small differences in the description of the intervention (e.g., $100 \mu \mathrm{l}$ versus $110 \mu \mathrm{l}$ of a drug), and in 49 pairs, partial agreement involved additional information about the intervention (e.g., dosage, time of administration, duration, etc.) in one source that was unavailable in the other. In 12 pairs, additional information was present only in the ClinicalTrials.gov record, and in 29 pairs additional information was present only in the abstract. For eight pairs, additional complementary information was present in both sources. Partial agreement also involved additional treatment arms: authors reported between one and four additional treatment arms in 11 ClinicalTrials. gov records and between one and six additional treatment arms in 25 abstracts.

\section{Masking or blinding}

We extracted information on masking separately for study participants, treatment administrators, and outcome assessors, finding that the ClinicalTrials.gov record frequently provided information on masking that was not available in the abstract (Figure 3).

\section{Sample size}

The number of study participants or sample size was reported in both the abstract and ClinicalTrials.gov for the majority $(136 / 154,88 \%)$ of studies. There were eight studies in which a sample size was reported in the trials

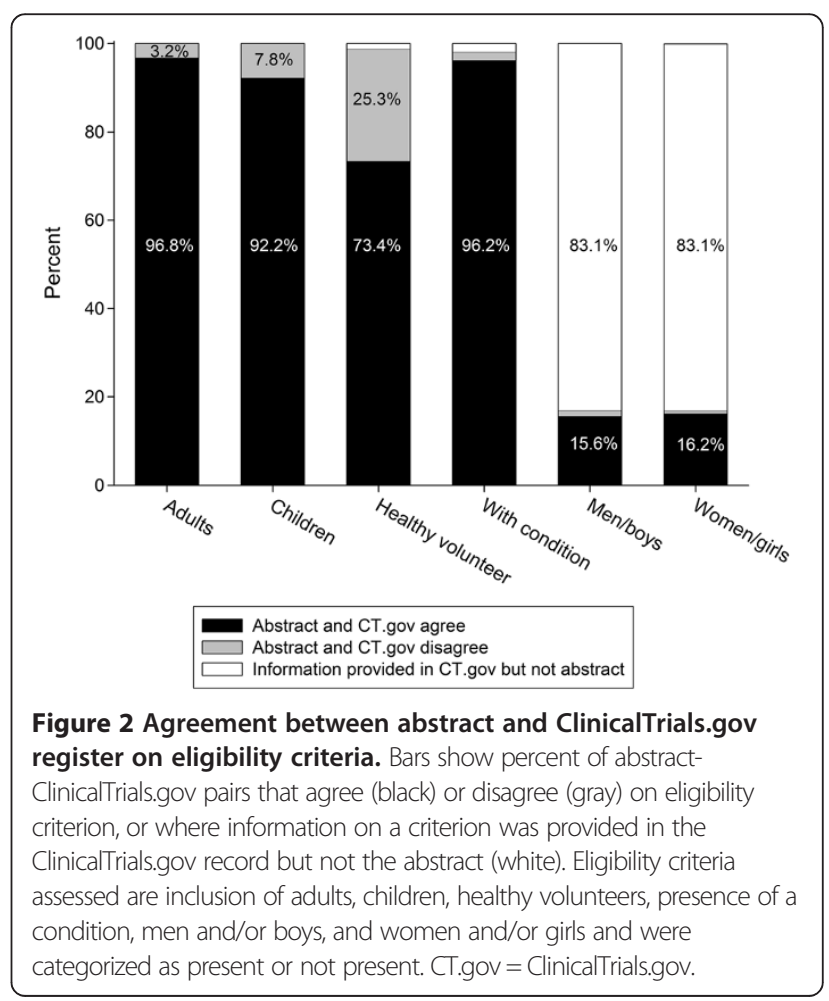


Table 2 All interventions reported in ClinicalTrials.gov register and abstract that were classified as disagreements

\begin{tabular}{|c|c|c|}
\hline & Abstract & ClinicalTrials.gov \\
\hline \multirow[t]{6}{*}{1} & $\begin{array}{l}\text { 1. lutein, zeaxanthin and long chain polyunsaturated fatty } \\
\text { acids (LCPUFAs) }\end{array}$ & 1. lutein, zeaxanthin, and 3 omega polyunsaturated fatty acid (PUFA) \\
\hline & 2. lutein and zeaxanthin & 2. placebo for 3 omega (PUFA) \\
\hline & 3. LCPUFAs, docosahexanoic acid, and eicosapentanoic & 3. placebo for lutein, zeaxanthin and 3 omega PUFA \\
\hline & & 4. $4 \mathrm{mg}$ triamcinolone \\
\hline & & 5. $1 \mathrm{mg}$ triamcinolone \\
\hline & & 6. standard of care \\
\hline \multirow[t]{6}{*}{2} & 1. $0.5 \mathrm{D}$ lens, head fixed & 1. spectacle lenses \\
\hline & 2. $0.5 \mathrm{D}$ lens, head free & 2. different spectacle lenses \\
\hline & 3. 1.0D lens, head fixed & \\
\hline & 4. $1.0 \mathrm{D}$ lens, head free & \\
\hline & 5. plano lens, head fixed & \\
\hline & 6. plano lens, head free & \\
\hline \multirow[t]{4}{*}{3} & 1. folic acid, vitamin B6 and vitamin B12 & 1. aspirin \pm vitamin $E$ \\
\hline & 2. placebo & 2. vitamin $\mathrm{E} \pm$ vitamin $\mathrm{C}$ \\
\hline & & 3. vitamin $\mathrm{E} \pm$ vitamin $\mathrm{C} \pm$ beta carotene \pm folate \\
\hline & & 4. placebo \\
\hline \multirow[t]{3}{*}{4} & 1. photocoagulation using ETDRS grid & 1. $810 \mathrm{~nm}$ diode laser photocoagulation \\
\hline & 2. photocoagulation with normal micro-pulsed technique & 2. argon laser photocoagulation \\
\hline & $\begin{array}{l}\text { 3. photocoagulation with high density micro-pulsed } \\
\text { technique }\end{array}$ & \\
\hline \multirow[t]{3}{*}{5} & 1. $0.3 \mathrm{mg}$ ranibizumab monthly for 6 months & $\begin{array}{l}\text { 1. treatment with ranibizumab until resolution of macular edema or as macular } \\
\text { edema occurs }\end{array}$ \\
\hline & 2. $0.5 \mathrm{mg}$ ranibizumab monthly for 6 months & \multirow{2}{*}{$\begin{array}{l}\text { 2. treatment with ranibizumab until resolution of edema and pigment epithelia } \\
\text { detachment or as macular edema or pigment epithelial detachment occur }\end{array}$} \\
\hline & $\begin{array}{l}\text { 3. } 3 \text { doses of ranibizumab, then treatment until macular } \\
\text { fluid or pigment epithelial detachment absent }\end{array}$ & \\
\hline \multirow[t]{2}{*}{6} & 1. laser photocoagulation & 1. early treatment \\
\hline & 2. conventional treatment & 2. standard of care \\
\hline \multirow[t]{2}{*}{7} & 1. SofFlex IOL & 1. Acrysof Natural lens \\
\hline & 2. SofPort IOL & 2. AmoSensar Lens \\
\hline \multirow[t]{4}{*}{8} & 1. plano lens plus weekend atropine & 1. patching plus near activities at least one hour per day \\
\hline & 2. weekend atropine & 2. atropine plus near activities at least one hour per day \\
\hline & 3. full spectacle correction plus weekend atropine & \\
\hline & 4. 2 hours daily patching & \\
\hline \multirow[t]{3}{*}{9} & $\begin{array}{l}\text { 1. contact lens care system with polyquaternium- } 1 \text { and } \\
\text { MAPD }\end{array}$ & 1. silicone hydrogel contact lens \\
\hline & 2. contact lens care system with $\mathrm{PHMB}$ & 2. 1 XPMBH preserved MPS \\
\hline & & 3. 1XPolyguad/Aldox MPS \\
\hline
\end{tabular}

register, but not the abstract. We compared the reported sample sizes for pairs where the abstract author stated that the number of participants represented all randomized study participants and the status on the ClinicalTrials.gov record was either "completed" or "active, not recruiting" (see Table 3), and found good agreement.

\section{Outcomes}

Authors reported 800 outcomes in 152 abstracts; no outcomes were reported in 2 abstracts. Thirty four percent $(52 / 154)$ of abstracts and $82 \%(126 / 154)$ of ClinicalTrials. gov records explicitly described a primary outcome (Table 4). Of the 80 primary outcomes reported among the 40 abstract - ClinicalTrials.gov pairs, 14 (18\%) were 


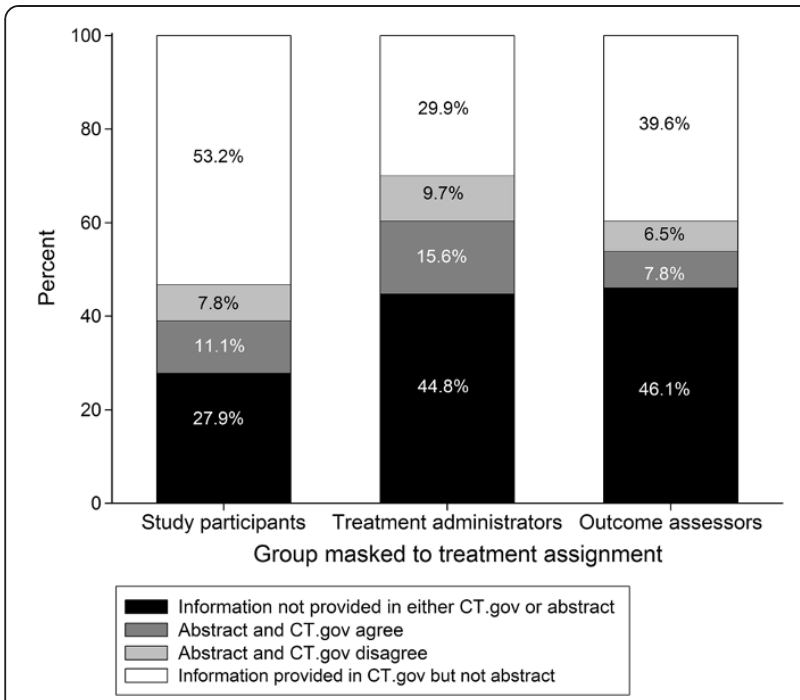

Figure 3 Agreement between abstract and ClinicalTrials.gov register on masking by study role. Bars show percent of abstractClinicalTrials.gov pairs that did not provide information on masking or blinding (black), agree (dark gray), or disagree (light gray), or where information was provided in the ClinicalTrials.gov record but not the conference abstract (white) on masking of study participants, persons administering the treatment, or persons measuring outcomes. Masking was categorized as present or not present. CT.gov = ClinicalTrials.gov

classified as being in complete agreement and 39 (49\%) as partial agreement (see Table 5). Partial agreement typically involved a more explicit description of an outcome as shown in in Table 6 . Of the remaining 27 primary outcomes reported in the abstract, 13 were reported elsewhere in the ClinicalTrials.gov record. There was poor agreement between non-primary outcomes reported in the abstract with those reported in ClinicalTrials.gov (see Table 5).

\section{Funders}

One hundred forty-six unique funders were identified in either the abstract or the ClinicalTrials.gov record. At

Table 3 Comparison of number of randomized study participants reported in abstract with number reported in ClinicalTrials.gov

\begin{tabular}{lc}
\hline Sample size comparisons for $\mathbf{6 6}$ abstract-ClinicalTrials.gov pairs \\
\hline No. (\%) pairs with exact match & $20(39)$ \\
No (\%) pairs with percent difference ${ }^{*} \pm 10 \%$ & $18(27)$ \\
No (\%) pairs with percent difference ${ }^{*}>10 \%$ & $28(42)$ \\
Range of percent differences & -106.6 to 88.7 \\
Average (SD) percent difference & $1.0(32.9)$ \\
\hline
\end{tabular}

*Percent difference $=(($ No. participants reported in abstract - No. reported in ClinicalTrials.gov)/ No. participants reported in ClinicalTrials.gov) X 100. Includes abstract-ClinicalTrials.gov pairs where number of participants in abstract included all randomized study participants and the status on the ClinicalTrials.gov record was either "completed" or "active, not recruiting.
Table 4 Agreement between conference abstract and ClinicalTrials.gov register on reporting of one or more primary outcomes ( $n=152$ abstract-Clinical Trials.gov pairs)

\begin{tabular}{lccc}
\hline Abstract & \multicolumn{3}{c}{ ClinicalTrials.gov } \\
\cline { 2 - 4 } & $\begin{array}{c}\text { Reporting } \geq \\
\text { primary } \\
\text { outcome } \\
\text { No. }\end{array}$ & $\begin{array}{c}\text { Not reporting a } \\
\text { primary outcome }\end{array}$ & $\begin{array}{c}\text { Total } \\
\text { number of } \\
\text { pairs }\end{array}$ \\
\hline $\begin{array}{l}\text { Reporting } \geq \text { one } \\
\text { primary outcome }\end{array}$ & 40 & No. & No. \\
$\begin{array}{l}\text { Not reporting a } \\
\text { primary outcome }\end{array}$ & 86 & 12 & 52 \\
$\begin{array}{l}\text { Total number of } \\
\text { pairs }\end{array}$ & 126 & 16 & 102 \\
\hline
\end{tabular}

least one funder was reported in each ClinicalTrials.gov record, but only in $62 \%$ of abstracts (95/154). More than one funder was reported in 41 ClinicalTrials.gov records and in 19 abstracts. The same funder(s) were reported in both sources for 10 studies. We observed partial agreement across funders for 37 pairs, in which an additional or different funder was reported in either the abstract or in the ClinicalTrials.gov record.

\section{Contact information}

The name of a contact person was included on $83 \%$ $(128 / 154)$ of ClinicalTrials.gov records, but a phone number was found on only 25 , and an email address on 26 records. No contact information was provided on any conference abstract except author name and affiliation.

\section{Discussion}

A substantial amount of additional information on study design was available on the ClinicalTrials.gov record that was not presented in a corresponding conference abstract. Information on multi-center status, eligibility criteria with respect to sex, who is masked, and primary outcome is

Table 5 Agreement between conference abstract and ClinicalTrials.gov register on outcomes ( $n=40$ abstractClinicalTrials.gov pairs reporting $\geq 1$ primary outcome and 152 abstract-clinicaltrials.gov pairs reporting a non-primary outcome)

\begin{tabular}{lcc}
\hline & $\begin{array}{c}\text { Primary } \\
\text { Outcome } \\
\text { No. (\%) }\end{array}$ & $\begin{array}{c}\text { Non-primary } \\
\text { outcome } \\
\text { No. (\%) }\end{array}$ \\
\hline $\begin{array}{l}\text { Total outcomes reported in } \\
\text { abstract }\end{array}$ & $80(100)$ & $708(100)$ \\
$\begin{array}{l}\text { Complete agreement } \\
\text { Partial agreement }\end{array}$ & $14(18)$ & $57(8)$ \\
$\begin{array}{l}\text { Not reported in ClinicalTrials. } \\
\text { gov }\end{array}$ & $39(49)$ & $205(29)$ \\
\hline
\end{tabular}




\begin{tabular}{|c|c|c|c|}
\hline & Abstract & ClinicalTrials.gov & Classified as \\
\hline 1 & $\begin{array}{l}\text { Retinal thickness in the peak oedematous field on the retinal } \\
\text { maps of the fast module scans of the Stratus OCT }\end{array}$ & $\begin{array}{l}\text { Retinal thickness by fast retinal thickness mapping by optical } \\
\text { coherence tomography at } 0,2,4,8,10,15,20,30,60,90,120 \text {,and } \\
180 \text { min after last swallow of glycerol }\end{array}$ & $\begin{array}{l}\text { Extra information in } \\
\text { ClinicalTrials.gov }\end{array}$ \\
\hline 2 & Visual acuity & $\begin{array}{l}\text { Mean change in best corrected visual acuity as assessed by } \\
\text { number letters read correctly on ETDRS eye chart at starting } \\
\text { test distance of } 4 \text { meters from baseline to } 1,3,6 \text { months }\end{array}$ & $\begin{array}{l}\text { Extra information in } \\
\text { ClinicalTrials.gov }\end{array}$ \\
\hline 3 & $\begin{array}{l}\text { ETDRS best corrected visual acuity at 2, } 6 \text { and } 12 \text { weeks; OCT } \\
\text { at } 2 \text { and } 6 \text { weeks; fluorescein angiography at } 2 \text { and } 6 \text { weeks }\end{array}$ & $\begin{array}{l}\text { Best corrected visual acuity, OCT foveal thickness, fluorescein } \\
\text { angiogram }\end{array}$ & $\begin{array}{l}\text { Extra information in } \\
\text { abstract }\end{array}$ \\
\hline 4 & $\begin{array}{l}\text { Change from baseline in major symptom complex score } \\
\text { over period } 1 \text {, consisting of runny nose, sniffles, itchy nose, } \\
\text { nose blows, sneezes, and watery eyes }\end{array}$ & $\begin{array}{l}\text { Mean change from baseline of major symptoms related to } \\
\text { seasonal allergic rhinitis }\end{array}$ & $\begin{array}{l}\text { Extra information in } \\
\text { abstract }\end{array}$ \\
\hline 5 & $\begin{array}{l}\text { Retinal detachment rate; surgery-related complications; } \\
\text { patient comfort; refractive change; visual acuity; OCT }\end{array}$ & Retinal detachment rate at 6 months & $\begin{array}{l}\text { Extra } \\
\text { complementary } \\
\text { information in both } \\
\text { sources }\end{array}$ \\
\hline
\end{tabular}

present to varying degrees in the ClinicalTrials.gov register record. In addition, the name of a contact was included in $83 \%$ of ClinicalTrials.gov records, so that if desired information about the trial was not available, or conflicted with what was reported in the abstract, a systematic reviewer could contact the study author directly, although contact information was provided in a minority of ClinicalTrials. gov records.

Thus, if a trial registration number is available for a study to be included in a systematic review, a systematic reviewer may be able to find additional information about study design in a trials register record. However, there are caveats to these findings. First, information about trial registration may not be required nor provided by conference organizers generally. Second, we encountered numerous disagreements between the information provided in the conference abstract with information contained within the ClinicalTrials.gov record on a number of items. ClinicalTrials.gov was originally intended to provide a source of information about the existence of a trial for patients and clinicians, to diminish redundant research effort, and to alert researchers to the possibility of publication bias. Currently it provides limited protocol information, although it would be a natural repository for full protocols. A published protocol or design and methods paper would provide more information, but until public availability of all trial protocols is achieved, trials registers serve as surrogates for study protocols.

The findings from our study relate specifically to ARVO abstracts and ClinicalTrials.gov and may not be applicable to other conference abstracts or trials registers. Furthermore, our requirement that a valid ClinicalTrials.gov registration number be reported on the abstract submission form could mean that studies were actually registered but the number not recorded or incorrectly recorded.

Previous studies have identified discrepancies between trials register records and associated full length publication on study design characteristics, especially related to the description of primary and secondary outcomes [11-16]. In our study, we found discrepancies related to the detailed descriptions of study interventions and outcomes reported in the conference abstract compared with those reported in the ClinicalTrials.gov record. Frequently there was more information available in the abstract than was in the ClinicalTrials.gov record (e.g., dose or duration of treatment). Most disturbing was the appearance of additional treatment arms in the abstract that were not included in the register, suggesting that investigators are not regularly updating the trial register record as required or that abstracts represent preliminary findings, before an arm was dropped.

The discrepancies in outcomes may be an indication of error, selective outcome reporting, or simply due to investigators not updating the ClinicalTrials.gov record in a timely manner. We obtained register records that were available shortly following presentation of abstract results at ARVO, so any additional outcomes or changes in the study design should have been incorporated into the trial register as an amendment to the protocol items. In addition, we compared outcomes in only one direction, i.e. from abstract to Clinicaltrials.gov record. We did not do the reverse analysis (i.e. comparing all outcomes reported in ClinicalTrials.gov with those reported in the abstract) because we would not expect an abstract to include all outcomes reported in ClinicalTrials.gov. In so doing, we did find that sometimes the outcome reported in the abstract as the "primary" outcomes was a secondary outcome in the ClinicalTrials.gov record. 
In the future it will be of interest to compare outcomes reported as results with the specified outcomes in the ClinicalTrials.gov record. Results reporting in ClinicalTrials.gov opened in September 2008. When we retrieved the records in May and June 2009, none of the records included any study results when downloaded.

Other studies have also found discrepancies between the description of an RCT as reported in an abstract with what was reported in a full length publication [17-21]. It would be of interest to make a three-way comparison of a conference abstract, trial register and results record, and full length publication to determine congruence between these sources of information. It would also be useful for authors to include conference abstracts in the ClinicalTrials.gov record as a publication, in addition to full length publications.

Whether the study results reported in an abstract should be included in a systematic review or used to make clinical decisions is unclear. That results from abstracts are being included in systematic reviews has been reported in a sample of meta-analyses indexed in Medline [7], for health technology assessments [5], and for drug formulary decision-making [22]. Including abstracts in systematic reviews is recommended by IOM [1], the Cochrane Collaboration [23], and the Agency for Health Care Research and Quality [24], although caution is urged due to the preliminary nature of abstract results $[25,26]$. Results from a Cochrane systematic review found that there was an overall smaller treatment effect if systematic reviewers included abstract results in the review compared to the treatment effect size without abstracts included, although this finding was not statistically significant [3]. Additional investigators report similar results, showing small reductions in the size of the treatment effect when abstract results are included in a systematic review $[27,28]$.

Overall, given our findings, we would encourage systematic reviewers to take advantage of the information provided in the ClinicalTrials.gov record to supplement the information provided in an abstract. We did not record the amount of time it took to extract information for each record. Because the trials register number was available, matching the abstract to the registry report was straightforward. Extracting all the information we did for our study was somewhat time consuming, and we found that it was more efficient to obtain the information from the tabular view in ClinicalTrials.gov rather than the full text view. Most likely, it would take much less time for a systematic reviewer looking for specific information. We would caution systematic reviewers to exercise care in using data from a trials register record, however, as with any unpublished study result. In many cases, systematic reviewers may not be able to distinguish between conflicting reports and still find it necessary to contact the study investigators. For this situation, the ClinicalTrials.gov record frequently provides contact information that is almost always missing from a conference abstract.

\section{Conclusions}

Systematic reviewers may find additional information about an RCT in the ClinicalTrials.gov record to supplement the information provided in a conference abstract. However, it may still be necessary to contact study investigators for information not included in either the abstract or ClinicalTrials.gov record, or that is in conflict between the two sources.

\section{Abbreviations}

ARVO: Association for Research in Vision and Ophthalmology;

CONSORT: Consolidated Standards of Reporting Trials; IOM: Institute of

Medicine; RCT: Randomized controlled trial.

\section{Competing interests}

The authors declare that they have no competing interests.

\section{Authors' contributions}

RWS conceived of the study, participated in the study design and data extraction, entered the data in the Access database, participated in the analysis and interpretation of results, and drafted the manuscript. LH participated in the study design, data extraction, analysis and interpretation of results. AE participated in the study design, data extraction, and interpretation of results. JT participated in the study design and data extraction. KD participated in data extraction and interpretation of results. All authors read, edited, and approved the final manuscript.

\section{Funding}

Funding for this study was provided by the National Eye Institute, National Institutes of Health, Grant 1U01EY020522. Publication of this article was funded in part of the Open Access Promotion Fund of the Johns Hopkins University Libraries.

\section{Author details}

${ }^{1}$ Center for Clinical Trials, Department of Epidemiology, Johns Hopkins Bloomberg School of Public Health, Baltimore, MD 21205, USA. 'Baptist Desoto Hospital, Southaven, MS 38671, USA.

Received: 1 February 2013 Accepted: 7 June 2013

Published: 18 June 2013

\section{References}

1. IOM (Institute of Medicine): Knowing What Works in Health Care: a Roadmap for the Nation. Committee on Reviewing Evidence to Identify Highly Effective Clinical Services. Washington DC: The National Academic Press; 2011.

2. Scherer RW, Langenberg P, Von Elm E: Full publication of results initially presented in abstracts. Cochrane Database Syst Rev 2007, 2. doi:10.1002/ 14651858.MR000005.pub3. Art. No.: MR000005.

3. Hopewell S, McDonald S, Clarke MJ, Egger M: Grey literature in metaanalyses of randomized trials of health care interventions. Cochrane Database Syst Rev 2007, 2. Art. No.:MR000010. DOl: 10.1002/14651858. MR000010.pub3.

4. Hopewell S, Eisinga A, Clarke M: Better reporting of randomized trials in biomedical journal and conference abstracts. J Information Science 2008, 34:162-173.

5. Dundar Y, Dodd S, Williamson P, Walley T, Dickson R: Searching for and use of conference abstracts in health technology assessments: Policy and practice. Int J Technol Assess Health Care 2006, 22:283-287.

6. Egger M, Juni P, Bartlett C, Holensteing F, Sterne J: How important are comprehensive literature searches and the assessment of trial quality in 
systematic reviews? Empirical study. Health Technol Assess 2003, 7(Issue 1):1-76.

7. Cook DJ, Guyatt GH, Ryan G, Clifton J, Buckingham L, Willan A, Mcllroy, Oxman AD: Should unpublished data be included in meta-analyses? JAMA 1993, 269:2749-2753.

8. Association for Research in Vision and Ophthalmology Statement on Registering Clinical Trials: http://www.arvo.org/About_ARVO/Policies/ Statement_on_Registering_Clinical_Trials.

9. Scherer RW, Sieving P, Dickersin K: Can we depend on investigators to identify and register randomized controlled trials? PLoS One 2012, 7: e44183. doi:10.1371/journal.pone.0044183.

10. Training Manual for Handsearchers, US Cochrane Center: [http://us. cochrane.org/sites/us.cochrane.org/files/uploads/pdf/Handsearcher\% 20Training\%20Manual.pdf].

11. Dwan K, Altman DG, Cresswell L, Blundell M, Gamble CL, Williamson PR: Comparison of protocols and registry entries to published reports for randomised controlled trials. Cochrane Database Syst Rev 2011 (Issue 1). Art. No.: MR000031. DOI: 10.1002/14651858.MR000031.pub2.

12. Ghandi R, Jan M, Smith HN, Mahomed NN, Bhandari M: Comparison of published orthopaedic trauma trials following registration in Clinicaltrials.gov. BMC Musculoskelet Disord 2011, 12:278. At. http:// biomedcentral.com/1471-2474/12/278.

13. You B, Gan HK, Pnd G, Chen EX: Consistency in the analysis and reporting of primary end points in oncology randomized controlled trials form registration to publication: a systematic review. J Clin Oncol 2012, 30:210-216.

14. Mathieu S, Boutron I, Moher D, Altman DG, Ravaud P: Comparison of registered and published primary outcomes in randomized controlled trials. JAMA 2009, 302:977-984.

15. Millette K, Roseman M, Thombs BD: Transparency of outcome reporting and trial registration of randomized controlled trials in top psychosomatic and behavioral health journals: a systematic review. $J$ Psychosomatic Research. 2011, 70:205-217.

16. Bourgeois F, Murthy S, Mandl KD: Outcome reporting among drug trials registered in ClinicalTrials.gov. Ann Intern Med 2010, 153:158-166.

17. Toma M, McAlister FA, Bialy L, Adams D, Vendermeer B, Armstrong PW: Transition from meeting abstract to full-length journal article for randomized controlled trials. JAMA 2006, 295:1281-1287.

18. Dundar Y, Dodd S, Williamson P, Dickson R, Walley T: Case study of the comparison of data from conference abstracts and full-text articles in health technology assessment of rapidly evolving technologies: Does it make a difference? Int J Tech Assess Health Care 2006, 22:288-294.

19. Prasad S, Lee DJ, Yuan JC-C, Barao VAR, Shyamsundeer N, Sukotjo C: Discrepancies between abstracts presented at International Association for Dental Research annual sessions from 2004 to 2005 and full-text publications. International J Dentistry 2012(859561). doi:10.1155/2012/859561.

20. Tam VC, Hotte SJ: Consistency of phase III clinical trial abstracts presented at an annual meeting of the American Society of Clinical Oncology compared with their subsequent full-text publications. J Clin Oncol 2008, 26:2205-2211.

21. Snedeker KG, Campbell M, Totton SC, Guthrie A, Sargeant JM: Comparison of outcomes and other variables between conference abstracts and subsequent peer-reviewed papers involving pre-harvest or abbatoir-level interventions against foodborne pathogens. Prev Vet Med 2010, 9:67-76.

22. Weizman AV, Griesman J, Bell CM: The use of research abstracts in formulary decision making by the Joint Oncology Drug Review of Canada. Appl Health Econ Policy 2010, 8:387-391.

23. Cochrane Handbook of Systematic Reviews of Interventions: [http:// handbook.cochrane.org/].

24. Relevo R, Balshem H: Finding evidence for comparing medical interventions: AHRQ and the Effective Health Care Program. J Clin Epidem 2011, 64:168-1177.

25. Falagas ME, Rosmarakis ES: Clinical decision-making based on findings presented in conference abstracts: is it safe for our patients? Eur Heart $J$ 2006, 27:2038-2039.

26. Wong SS-W, Fraser C, Lurenco T, Barnett D, Avenell A, Glazener C, Cuthberston B, N'Dow J: The fate of conference abstracts: systematic review and meta-analysis of surgical treatments for men with benign prostatic enlargement. World J Urol 2010, 28:63-69.

27. Hoehner C, Soares J, Parra DC, Ribeiro IC, Pratt M, Bracco M, Hallal PC, Bownson RC: Physical activity interventions in Latin America: What value might be added by including conference abstracts in a literature review? J Physical Activity and Health 2010, 7(Suppl 2):S265-S278.

28. Hopewell S, Clarke M, Moher D, Wager E, Middleton P, Altman DG, Schulz KF, CONSORT Group: CONSORT for reporting randomized controlled trials in journal and conference abstracts: Explanation and elaboration. PLOS Med 2008, 5:e20. doi:10.1371/journal.pmed.0050020.

doi:10.1186/1471-2288-13-79

Cite this article as: Scherer et al:: ClinicalTrials.gov registration can supplement information in abstracts for systematic reviews: a comparison study. BMC Medical Research Methodology 2013 13:79.

\section{Submit your next manuscript to BioMed Central and take full advantage of:}

- Convenient online submission

- Thorough peer review

- No space constraints or color figure charges

- Immediate publication on acceptance

- Inclusion in PubMed, CAS, Scopus and Google Scholar

- Research which is freely available for redistribution

Submit your manuscript at www.biomedcentral.com/submit
C Biomed Central 